\title{
Reflets
}

Revue d'intervention sociale et communautaire

\section{Une lutte constante pour contrer les violences faites aux femmes et leur assurer des services en français de qualité} Entrevue avec Ghislaine Sirois

\section{Marie-Luce Garceau, Sébastien Savard et Stéphane Richard}

Volume 18, numéro 1, printemps 2012

L'intervention communautaire chez les francophones minoritaires : des pratiques à découvrir

URI : https://id.erudit.org/iderudit/1012330ar

DOI : https://doi.org/10.7202/1012330ar

Aller au sommaire du numéro

Éditeur(s)

Reflets, Revue d'intervention sociale et communautaire

ISSN

1203-4576 (imprimé)

1712-8498 (numérique)

Découvrir la revue

Citer ce document

Garceau, M.-L., Savard, S. \& Richard, S. (2012). Une lutte constante pour contrer les violences faites aux femmes et leur assurer des services en français de qualité : entrevue avec Ghislaine Sirois. Reflets, 18(1), 22-37.

https://doi.org/10.7202/1012330ar 


\section{Une lutte constante pour contrer les violences faites aux femmes et leur assurer des services en français de qualité}

\section{Entrevue avec Ghislaine Sirois}

Ghislaine Sirois est directrice générale d'Action ontarienne contre la violence faite aux femmes, Ottawa, Ontario

L'entrevue a été réalisée par Marie-Luce Garceau, Sébastien Savard et Stéphane Richard

Les grands principes qui animent le travail de madame Ghislaine Sirois sont nombreux. Dans les domaines de l'intervention communautaire et du changement social, son travail se caractérise par la liberté de parole et d'examen, le dialogue ouvert et rationnel ainsi que la probité intellectuelle et éthique.Au cours des années, Action ontarienne contre la violence faite aux femmes $(\mathrm{AOcVF})^{1}$ a su développer, en collaboration étroite avec d'autres collègues et groupes sociaux, de nombreux partenariats qui ont mené au développement de recherches et surtout au développement de nouveaux services en français en matière de violence faite aux femmes. Tous ces partenariats ont été établis dans le respect des partenaires, et tous sont marqués par l'importance que madame Sirois accorde à la responsabilité sociale des partenaires et au développement des collectivités afin qu'elles puissent elles-mêmes répondre aux besoins des femmes et de la société. Elle a réussi à créer des liens étroits et solides avec les organismes sociaux et communautaires et avec plusieurs ministères et institutions. Depuis des années, madame Sirois travaille souvent dans l'ombre; pourtant, 
elle influence beaucoup le bien-être des femmes et, par ricochet, celui de leur famille, et ce, quel que soit le coin de la province où ces femmes habitent. Finalement, ce qui caractérise madame Sirois, c'est qu'elle croit fermement en la capacité des femmes et des hommes d'aujourd'hui ainsi que des groupes sociaux de se prendre en main afin d'améliorer leur bien-être et de vivre dans une société plus juste et égalitaire.

M.-L. Garceau: Pouvez-vous expliquer ce que vous entendez par "intervention communautaire »?

G. Sirois : En fait, il faut remonter dans le temps, lorsque j'étais bénévole dans un centre d'aide et de lutte contre les agressions à caractère sexuel. Alors, tout était à faire... Ç’a été une bonne école. C'est à cette époque que j'ai acquis les fondements de l'intervention féministe auprès des femmes, mais aussi que l'on a beaucoup discuté sur le changement social et sur les moyens à utiliser pour éliminer la violence envers les femmes.

Depuis mon arrivée à AOcVF, c'est le concept de la « reprise de pouvoir " qui est à l'origine des actions proposées. En effet, les femmes francophones de l'Ontario se sont souvent senties exclues du "mouvement des femmes ", car on n'y comprenait pas pourquoi la langue et la culture prenaient une aussi grande place; comme si elles étaient des éléments secondaires à la « lutte». On peut aisément comprendre les raisons pour lesquelles les femmes immigrantes, les femmes noires et les femmes autochtones ont pu aussi se sentir " exclues » du mouvement, car on prêtait peu oreille à l'intersectionnalité des oppressions, à l'impact cumulatif des différentes formes de discrimination vécues par les femmes.

Je me souviens avoir participé à une rencontre à Toronto, vers 1992 ou 1993. Les femmes francophones du réseau m'y ont envoyée en disant «Vas-y! C'est à ton tour! » Naïve, j'y suis allée, pour : 1. me faire engueuler copieusement quand j'ai osé parler de services en français, et 2. constater à quel point nous étions coupées des informations qui circulaient sur les enjeux, les projets de loi, les développements des programmes, etc. À l'époque, nous avions peu d'alliées, et nous n'avions pas accès à l'information qui nous 
permettrait d'avoir le sentiment d'avoir une emprise sur les défis à relever. Je crois que notre réseau était en mode survie, non seulement à cause du financement inadéquat, mais aussi à cause du peu de pouvoir qu'il avait sur l'ensemble des situations qui touchent les femmes : les tribunaux, les programmes, l'accès aux ressources, l'accès aux décideurs, etc. On avait du travail à faire!

L'intervention communautaire consiste donc à faire en premier lieu des constats sur les besoins et les défis rencontrés. Dans cette tâche, on a eu de l'aide de chercheuses universitaires et communautaires $^{2}$ qui ont travaillé avec nous pour nous aider à documenter, à interpréter les données, à développer les principes qui sous-tendent notre action, à nous informer sur les enjeux, à rêver à ce qu'on voudrait, à planifier notre action de manière concertée.

"Concertation », le mot magique! Il était nécessaire de s'entendre sur les priorités, les besoins, les moyens, et les messages à passer. Par la suite, il était important d'accepter de travailler en partenariat, de mettre sur la glace les besoins de son organisme pour défendre les priorités ailleurs. Le soutien mutuel, qui sur le plan individuel peut jouer un rôle important dans la vie des femmes, ça se pratique aussi sur le plan collectif. Selon moi, il n'y a pas de meilleur exemple que le rôle qu'a joué Gaëtanne Pharand ${ }^{3}$ du Centre Victoria pour femmes de Sudbury. Elle a joué un rôle déterminant dans le développement des services en français (SEF) en matière de violence contre les femmes en Ontario par sa participation à une multitude de rencontres. Elle a aussi soutenu au quotidien le développement des services en français à Timmins et dans le comté de Simcoe. À son grand altruisme personnel s'ajoute une conviction profonde à l'égard des SEF qui fait d'elle une leader remarquable.

Comme le soutien mutuel, la reprise du pouvoir des femmes s'exerce sur le plan non seulement individuel, mais aussi collectif. Une femme victime de violence, on lui a enlevé son pouvoir, par la force, le chantage, le contrôle, les menaces, mais aussi en l'ignorant, en ignorant ses besoins, ses aspirations... C'est un peu ce qui se passait pour la communauté des femmes francophones, jusqu'à ce 
que la Loi sur les services en français (LSEF) ne soit mise en œuvre. Là, les décideurs ont cherché à savoir pas nécessairement ce que voulaient les femmes, mais comment les ministères pouvaient répondre à leurs obligations sous la LSEF. À vrai dire, je ne crois pas que quiconque savait à ce moment-là de quelle manière pouvait être utilisée la LSEF pour mieux servir les francophones.

Il fallait donc savoir ce que nous voulions et proposer les moyens d'y arriver. Ça s'est fait dans le concret, à partir d'initiatives mises en place au cours des années : ce qui marche, ce qui ne marche pas, ce qui est un vrai SEF, ce que ça veut dire d'utiliser une approche féministe. On a osé démarrer des services avec des petites sommes, et là où les femmes francophones ou francophiles assumaient la gouvernance, les services ont pris de l'expansion. Ce constat a été l'une des premières clés du développement des SEF à l'intention des femmes victimes de violence : nous avions à (re)prendre le pouvoir sur les services qui devaient nous desservir, à les diriger, à les mettre à notre image, à les défendre.

Nous avons dès le départ mis en place des rencontres régulières des intervenantes et des directrices des SEF, et ce sont ces rencontres qui ont permis d'identifier les besoins, les moyens, etc. Mais ce sont aussi ces rencontres qui nous ont permis d'analyser notre situation collective, de faire des constats - comme celui sur la gouvernance - , de savoir dans quelle direction aller pour assurer le développement de nos services et d'apprendre ensemble à revendiquer auprès des décideurs politiques et administratifs.

M.-L. Garceau: Quelles sont les personnes ou les idées qui vous ont marquée ou influencée dans le domaine de l'intervention communautaire? D'où vous est venu ce désir d'engagement dans la communauté?

G. Sirois : J'ai déjà nommé Gaëtanne, pour sa conviction envers les SEF à l'intention des femmes. Je pense aussi à Suzanne Cloutier du Service familial catholique pour ses apports théoriques sur l'intervention, en particulier sur le soutien mutuel.

J'ai eu plusieurs mentors au cours de mon cheminement féministe : les femmes de la collective du CALACS où j'étais en 1979; les survivantes dans les groupes de soutien; les jeunes filles que 
j'ai rencontrées dans les classes où je faisais de la sensibilisation communautaire, et bien d'autres encore. Toutes, elles m'ont aidée à concrétiser les idées discutées dans mes premières fréquentations féministes, faites d'abord au moyen de lectures : Annie Leclerc, Benoîte Groult, Simone de Beauvoir... puis les Américaines, Andrea Dworkin, Kate Millet, Adrienne Rich et d'autres.

Les femmes immigrantes impliquées dans le réseau m’ont beaucoup appris. Elles apportent des énergies neuves, des nouvelles réalités. Souvent, elles n'ont pas vécu l'oppression liée à la langue de la même façon que les Franco-ontariennes dites «de souche " l'ont vécue. Ainsi, pour elles, réclamer des services en français, ça ne les intimide pas du tout! Elles n'ont pas, pour la plupart, intériorisé cette oppression liée à la langue comme certaines d'entre nous l'ont fait. Elles ont vécu de nombreuses oppressions par contre, et lorsque nous pouvons faire le lien, comme femmes, entre les différentes formes d'oppression et sur la façon dont nous les intériorisons, nous savons alors que nous avons bien plus en commun que de différences! En agissant en solidarité, nous sommes toutes plus fortes et savantes.

Pour moi, l'intervention communautaire est venue du désir d'agir et de changer le monde, d'en faire un endroit qui appartient aussi aux femmes. Je souhaitais que cessent cette abjecte violence contre les femmes, les viols qui détruisent les esprits des femmes et des filles, les féminicides, l'exploitation, la discrimination. Je souhaitais que cesse l'exclusion à cause du genre ou des habiletés, de l'orientation sexuelle, de la couleur de la peau. À cette période de ma vie,j'ai lu Paolo Freire, son livre sur la Pédagogie des opprimés, et plein de lumières se sont allumées... La trajectoire était tracée.

M.-L. Garceau: Si vous aviez à nommer les défis les plus importants qui traversent le mouvement communautaire et les communautés francophones en Ontario, quels seraient-ils?

G. Sirois : Je nommerais en premier lieu la montée du conservatisme tant sur le plan politique que religieux. Sur le plan politique, car je crois qu'il y a un sérieux revirement de situation au Canada par rapport à l'égalité non seulement des femmes, mais des individus 
et des groupes qui pourraient profiter de plus de justice sociale, qui réclament un environnement sain, des chances égales en matière d'éducation et de soins de santé.

Ce mouvement politique est soutenu par un puissant conservatisme religieux qui n'existe pas juste au Canada, et dont on voit l'influence, par exemple, dans le processus actuel du choix du candidat républicain aux prochaines élections à la présidence. Ces groupes de lobby, qui sont contre les droits des gais et lesbiennes ou contre le droit à l'avortement, par exemple, sont également présents dans les bureaux des députés et ministres conservateurs canadiens, et c'est inquiétant. Il s'agit non seulement d'un conservatisme religieux, mais il est aussi chrétien et fondamentaliste, ce qui pourrait nous mener tout droit à l'islamophobie ou l'antisémitisme. Il faut le reconnaitre, ces trois grandes religions n'ont pas toujours été tendres à l'égard des femmes et le conservatisme religieux de tout acabit est une menace aux droits à l'égalité des femmes.

Le conservatisme politique et moraliste porte aussi atteinte aux droits à la sécurité des femmes - on pense à l'élimination du registre des armes à feu, par exemple — et aux droits fondamentaux des jeunes délinquants, par exemple. Les personnes pauvres - et on sait que bien des femmes sont pauvres — sont aussi affectées : les changements qui s'annoncent au régime universel de santé - une privatisation accrue - nuiront à leur accès à des soins de santé. Les allocations pour des logements adéquats et abordables sont désastreuses, on rencontre de plus en plus de sans-abri dans un pays où la température descend à moins 30 ou 40 ! Qu'en est-il des droits sociaux et économiques? Des droits à la sécurité? À la même protection de la loi? Au Canada, avons-nous besoin de construire plus de prisons ou avons-nous besoin de plus de programmes pour mettre fin à la pauvreté et aux iniquités sociales?

En matière de violence contre les femmes, une approche punitive au détriment d'une approche qui vise l'atteinte des droits à l'égalité des femmes ne fonctionnera pas. Les peines plus sévères ne sont pas utiles, car en fin de compte, très peu d'hommes violents sont accusés et condamnés. Ces peines plus sévères ne donnent finalement qu'un faux sentiment de sécurité. Les femmes ont 
besoin d'indépendance économique pour pouvoir se sortir de situations violentes : avoir un emploi qui offre un salaire juste, avoir accès pour toutes à des garderies abordables, avoir un revenu de retraite ou des prestations d'aide sociale qui ne les condamnent pas à la pauvreté. Elles ont besoin d'être représentées à la cour de droit de la famille pour être en mesure de contrer les messages de la droite sur les questions de garde légale. Elles ont besoin d'être soutenues par la recherche qu'effectuent les groupes de femmes qui analysent les lois et les programmes du gouvernement afin de faire valoir les droits des femmes.

De plus, on entend davantage de messages d'intolérance à l'égard de la francophonie au Canada, et c'est le premier ministre Harper lui-même qui déclarait, alors qu'il était membre d'une coalition de droite, que le Canada n'est pas un pays bilingue, et qu'à part le Québec, le reste du Canada "is English speaking». Les gestes posés par le gouvernement canadien au cours des derniers mois : nomination d'un vérificateur et de juges de la Cour suprême unilingues anglophones, élimination de plus de 150 postes d'enseignement du français dans la fonction publique, tout ça, ça s'en va dans la direction d'un Canada unilingue anglophone hors Québec. L'Institut Fraser s'en est mêlé en dénonçant les coûts liés au bilinguisme et sous peu, Statistique Canada publiera un nouveau rapport, rédigé depuis les importants changements apportés aux formulaires de collectes de données, et il semble que la place des francophones pourrait s'en trouver diminuée. Ensemble, tout ça constitue une menace grandissante pour les minorités francophones et un message non équivoque qui nourrit la francophobie comme cela s'est récemment manifesté à Cornwall, où des manifestants opposés à la politique de bilinguisme de l'hôpital tenaient des affiches « one flag, one language, one country "... Il n'est jamais temps pour les francophones de se reposer!

Politiquement, l'Ontario se démarque présentement par son approche plus progressiste à l'égard de plusieurs de ces enjeux. Il faut tout de même tenir compte des facteurs décrits ci-haut, car ils finiront par avoir un impact au niveau provincial. On 
l'entend lors d'élections, par exemple. Strictement, sur le plan du développement des services en français à l'intention des femmes, il manque des politiques pour le développement des SEF qui confirmeraient que les avancées réalisées au cours des vingt dernières années ne sont pas des accidents de parcours. Il importe aussi que le gouvernement provincial finance la concertation des groupes de femmes. Il faut que les femmes puissent se parler des enjeux - la décriminalisation de la prostitution, par exemple qu'elles en fassent l'analyse, qu'elles déterminent leur plan d'action collectif. Ce manque de concertation a mené à des divisions par le passé, à de la confusion peut-être au sujet de certains enjeux et à un manque de cohésion dans ce que nous devrions exiger des gouvernements provincial et fédéral.

Comme à l'AOcVF, nous avons fait de nos rencontres régulières une priorité et nous avons trouvé les moyens de les financer. Je crois que c'est pour cette raison que les femmes francophones ont pu faire progresser non seulement les services directs, mais également les outils pour mieux desservir les femmes comme la formation, le matériel en français, les analyses d'enjeux. L'intervention communautaire d'envergure doit compter sur ces petits groupes d'activistes en régions. Et ces activistes doivent être soutenues, outillées, avoir l'occasion d'en rencontrer d'autres qui ont les mêmes objectifs qu'elles.

M.-L. Garceau : D'après vous, est-il toujours possible pour le mouvement communautaire de faire pression sur l'État en faveur des exclus? Comment dériveriezvous la conjoncture politique pour le milieu communautaire en Ontario francophone?

G. Sirois : Comme je l'ai déjà mentionné, je crois que le leadership du gouvernement provincial en Ontario présentement est progressiste. Il est vrai qu'au sortir de huit ans d'un gouvernement Harris, les libéraux ont l'apparence de la gauche radicale! Depuis quelque temps, nous avons assisté à de bonnes décisions, par exemple en éducation, pour tenter de diminuer l'exclusion des enfants moins nantis. Il y a un programme d'éducation à la petite enfance, à temps plein, qui libère des places en garderie, places réclamées par les femmes depuis trente ans! Si malgré les augmentations 
récentes, les indemnités d'aide sociale sont encore trop basses, au moins il n'y a plus de harcèlement sous prétexte de " chasse aux fraudeurs ", ni de ligne téléphonique pour dénoncer son voisin ou sa conjointe prestataire.

Le gouvernement actuel a aussi eu de bonnes initiatives en matière de violence faite aux femmes : un plan d'action en matière de violence familiale et un plan d'action contre la violence à caractère sexuel. Ces plans comportaient des actions concrètes, pas toutes parfaites il est vrai, et des moyens concertés pour travailler à l'élimination de la violence conjugale et sexuelle à l'égard des femmes et des jeunes filles. Ce sont des plans d'action qui ont une analyse féministe, qui reflètent les résultats des consultations qui ont été menées, et qui font appel aux groupes communautaires, le plus souvent, pour en faire la mise en œuvre.

Je dirais donc que le climat politique actuel est favorable au niveau provincial, même s'il est, selon moi, désastreux au niveau fédéral. Avec le gouvernement actuel en Ontario,AOcVF a pu rencontrer des ministres pour vraiment parler des enjeux. Nous avons réussi à obtenir du financement pour des projets importants, comme la maison d'hébergement francophone de Timmins et celle de Toronto, l'Institut de formation des intervenantes, la campagne Voisin-es, ami-es et familles en français. Certes, il y a toujours une place à l'amélioration. Par exemple, dans la répartition de fonds pour les SEF dans le cadre de la nouvelle initiative de soutien juridique, là, à notre avis, le ministère fait fausse route. Si nous avions obtenu ces politiques de développement des SEF dont on parlait plus tôt, avec de bons critères pour déterminer lequel des services est le plus en mesure de garantir que l'offre active et continue de SEF, on ne serait pas là à contester ces dernières décisions et à demander qu'elles soient révisées.

Nous avons constaté que d'autres groupes francophones ont aussi profité du climat politique favorable des dernières années, par exemple, les aînés, les personnes handicapées, les jeunes. Il y a eu plus de place pour les initiatives en français issues du domaine communautaire. Tout n'est pas réglé, loin de là. Mais on sent un 
soutien concret, réel, de la part du gouvernement de l'Ontario depuis les huit dernières années.

M.-L. Garceau : D'après vous, peut-on parler d'un renouvellement de l'État social en Ontario?

G. Sirois : C'est si fragile! Lors de la prochaine élection, allons-nous revenir en arrière? Les progrès seront-ils effacés? De quelle façon peuton continuer à progresser socialement alors que nous sommes confrontés à une droite virulente, habile à vendre sa salade et qui ne fait aucun cas de l'opposition?

Je crois que le gouvernement actuel en Ontario a pris des engagements importants envers une société plus juste. Je crois que la seule façon de contrer le conservatisme absolutiste, c'est de (re)prendre le pouvoir en se servant de son droit de vote. Les femmes, les personnes exclues, les jeunes, toutes et tous doivent aller voter! Toutefois, nous devons faire une meilleure job afin d'expliquer pourquoi il est important d'aller voter.

Actuellement, du côté des conservateurs, on compte sur l'inertie de l'électorat, car leurs partisans, minoritaires au Canada il faut le dire, iront voter. Je crois que nous devons mieux mobiliser, informer, inciter celles et ceux qui ont le droit de voter et qui ne se retrouvent pas dans ces politiques et ce style de leadership.

Je sais, on pense : à quoi ça sert d'aller voter? Mais comme disait Winston Churchill, la démocratie n'est pas parfaite, mais c'est « le moins pire des systèmes " et nous devons nous faire entendre et nous opposer par notre vote, à ce qu'une minorité qui n'agit que dans ses propres intérêts soit maitresse de notre destinée!

M.-L. Garceau: Selon vous, quelle place occupe le mouvement communautaire pour le gouvernement de l'Ontario? Quel est son rôle? Quelle est son importance? Quelles sont ses responsabilités?

G. Sirois : Sur ce plan, il y a un changement important qui s'est dessiné au cours des dernières années. Je ne peux pas parler de tout le secteur communautaire, mais du secteur femmes où, pas qu'il y ait eu "désengagement de l'État », mais plutôt une passation des 
responsabilités au secteur communautaire, avec un financement de l'État. Je m'explique. Alors qu'auparavant, la Direction générale de la condition féminine de l'Ontario aurait élaboré une campagne de sensibilisation contre les agressions à caractère sexuel, dernièrement elle a confié au secteur communautaire - à la Ontario Coalition of Rape Crisis Centres et à AOcVF - la production des outils de base d'une telle campagne :messages clés, stratégies, etc. On pourrait se demander si un tel changement se produit parce que ça coûte moins cher à l'État. Je ne sais pas, mais ce que je sais, c'est que les personnes les plus habilitées à produire des outils efficaces sont autour de la table et prennent les décisions, à l'intérieur d'un cadre déterminé bien sûr, mais tout de même avec la latitude nécessaire pour que les messages soient féministes, approuvés par le plus grand nombre possible d'intervenantes dans les CALACS, tant francophones qu'anglophones.

Je crois qu'il y a beaucoup de potentiel dans ce genre de partenariat. Est-ce qu' on est "vendues »? Certains le diront peut-être, mais le leadership, ce n'est pas seulement de critiquer, parfois il faut prendre les rênes, au risque de se tromper. Si nous voulons que la société change dans le sens que nous le désirons, pour que les enjeux des femmes soient aussi sur la place publique, il faut s'impliquer, sauter sur la glace. Et parfois, aller dans les coins de patinoire.

Au cours des années, $\mathrm{AOcVF}$ a sauté sur la patinoire à bien des reprises, de même que ses groupes membres. Nous avions l'intuition que nous avions à montrer le chemin pour le développement des SEF, car ce n'était pas du tout clair, au niveau des ministères, la façon dont ce développement se ferait. Nous avons élaboré quatre plans d'action et nous les avons soumis aux ministères concernés, et ensemble nous avons réalisé bien des éléments de ces plans.

Je crois que c'est ça notre responsabilité : dénoncer le manque de SEF à l'intention des femmes, mais aussi proposer des moyens d'y remédier et plonger quand l'occasion nous est offerte de faire avancer les choses. Un exemple : le modèle de la ligne provinciale Fem'aide est un modèle unique, osé, qui tient compte 
des besoins régionaux et qui présente de nombreux défis sur le plan administratif. Est-ce que le modèle fonctionne bien? Oui, sans conteste. Est-ce que c'est facile? Non, pas toujours. Mais c'est notre modèle, il nous oblige à travailler en concertation, à discuter des approches, et je crois que les services obtenus à la ligne n'en sont que meilleurs.

Proposer des solutions, participer à la mise en œuvre des initiatives, ça revient à la " reprise de pouvoir ». Pour bien des femmes ayant connu des abus de pouvoir, juste l'idée de participer au pouvoir peut faire peur. Mais il y a des façons saines d'exercer le pouvoir, de le faire avec les autres et au bénéfice des autres, pas au détriment des autres.

M.-L. Garceau: Quels sont les acquis des organismes communautaires pour relever les défis du néolibéralisme?

G. Sirois : Certains « acquis " pourraient en fait ne pas être des « acquis »; ils pourraient être plus fragiles que d'autres. Il est possible, par exemple, qu'un organisme provincial comme AOcVF soit éliminé par un gouvernement moins favorable à la cause des femmes, mais il serait plus difficile d'éliminer les services directs comme ceux qu'offrent les maisons d'hébergement et les CALACS. C'est pourquoi les services directs se doivent d'être solides dans l'offre des services pour ne pas être vulnérables aux critiques.

Toutes, membres du conseil d'administration, directrices et intervenantes, doivent connaitre les enjeux liés au néoconservatisme : les risques de plus grande pauvreté pour les exclus - ne sommes-nous pas toutes et tous à une maladie ou un emploi près de l'exclusion? Il ne faut pas l'oublier! —; les risques pour la francophonie en milieu minoritaire; la privatisation des soins de santé; une approche moralisante en regard de la sécurité des femmes incluant l'accès à l'avortement sécuritaire... D'où l'importance de la formation et de la concertation. D'avoir documenté notre développement et de continuer à mettre sur papier ou en ligne les analyses féministes des enjeux qui porteraient atteinte aux droits à l'égalité et à la sécurité des femmes est important. 
Il faut s'échanger les informations, il faut s'inviter dans l'action; on ne doit pas baisser les bras et croire que tout est acquis pour toujours. Ce sont les groupes en régions, pour nous les CALACS, les maisons d'hébergement qui ont un mandat de changement social qui ont ce rôle à jouer. Les membres des conseils d'administration doivent être engagées, informées, déterminées. Les directrices et les intervenantes doivent comprendre les enjeux et participer à les faire connaitre dans les communautés. Il faut amener les femmes à voter, et pour ce, il faut qu'elles sachent que les enjeux qu'elles croient comme étant « réservés à ceux qui les comprennent mieux ", que ces enjeux sont les leurs : l'économie, la constitution - oui! oui! —, la justice, le désengagement de l'État, etc. Ce sont nos enjeux!

M.-L. Garceau: En fonction de votre champ d'expérience comme militante de longue date, quels sont les rapports actuels entre le mouvement féministe francophone et le gouvernement de l'Ontario?

G. Sirois : Je dirais que c'est un partenariat respectueux, sauf avec quelques instances qui n'ont pas encore compris la façon dont nous pouvons être utiles dans l'atteinte de leurs objectifs. Et je crois que la présence de femmes fortes au gouvernement - les ministres Meilleur, Matthews, Broten, Wynne et jadis Pupatello — et qui ont été soutenues par leur chef — les ministères dirigés par les femmes disposaient de $80 \%$ du budget total de la province avant les dernières élections - a fait une grande différence par rapport aux enjeux sociaux et nous espérons qu'elles seront écoutées au moment où sera déposé le rapport Drummond, et que les services sociaux ne seront pas trop écorchés.

Nous apprenons comment négocier ces partenariats, nous avons encore du travail à faire, en particulier dans le secteur justice, mais il est possible de travailler avec le gouvernement actuel et de réaliser certains de nos objectifs parmi les plus importants.

M.-L. Garceau: On reproche souvent au mouvement communautaire de s'être éloigné de sa base, de privilégier l'intervention individuelle à l'intervention communautaire et de faire plus de place aux professionnels qu'aux militants dans leur organisation. Comment réagissez-vous à cette critique? 
G. Sirois : Le mouvement ne pourrait survivre sans l'apport des bénévoles qui s'impliquent, en particulier dans les conseils d'administration. C'est sur leurs épaules que repose tout le réseau. Il est vrai que les "professionnelles » ont pris une place, mais on offre des services directs pour lesquels une formation pointue et continue est exigée, ainsi qu'une grande disponibilité qui est bien au-delà de ce que les bénévoles sont en mesure d'offrir bien souvent, à cause de leurs autres obligations. Sans compter les défis de recrutement, surtout dans les petites communautés francophones.

Les services aux femmes sont des services essentiels, à cause de l'inégalité entre les hommes et les femmes et le demeureront tant que l'égalité ne sera pas atteinte. Déjà, les écoles reposaient sur des bénévoles en quelque sorte, puisque ce sont les communautés religieuses qui ont créé les premières écoles dans le but de rendre l'éducation accessible. Les Sociétés d'aide à l'enfance ont été créées par des âmes charitables avant que leur rôle ne soit reconnu. Sommes-nous devant un phénomène semblable, où les services essentiels sexo-spécifiques liés à la violence subie par les femmes seront un jour assurés de survie?

Oui ça change la dynamique que de devenir un service «financé » plutôt que strictement militant et bénévole. Mais les femmes et les groupes de femmes ont aussi droit au financement de l'État pour répondre aux besoins des femmes victimes de violence. Ce sont aussi nos taxes!

Quand je pense aux argents versés pour des arénas professionnels, par exemple, pourquoi ne pas aussi penser aux infrastructures sociales comme aux garderies? Elles créent aussi des emplois et génèrent des revenus parce que les femmes qui peuvent ainsi aller travailler paient des taxes. Une étude au Québec a montré que le régime des garderies se payait quasi tout seul, grâce aux revenus générés par les femmes qui travaillent. Alors, pourquoi pas des services sociaux qui répondent à des besoins urgents des femmes?

L'intervention communautaire versus l'intervention individuelle? Oui, les services directs ont beaucoup à faire sur le plan 
individuel, c'est leur rôle premier. Je ne crois pas que nous ayons négligé l'intervention communautaire pour autant. Nous avons un programme de sensibilisation communautaire très proche du terrain, des milieux de travail et des groupes sociaux. Les organismes font des présentations dans la communauté, écrivent des lettres aux décideurs, participent aux débats affectant les femmes et la société en général. De plus et surtout, les groupes membres d'AOcVF ont été à l'avant-garde de l'offre des SEF en Ontario et ont réussi à faire valoir la pertinence de ces derniers "par et pour les femmes francophones ». Cette approche et ce slogan ont été maintes fois repris par d'autres secteurs, car il y avait un précédent.

L'intervention communautaire se fait peut-être différemment qu'il y a vingt ans, mais elle est toujours présente.

M.-L. Garceau: En bout de piste, comment les principes et les pratiques du mouvement contre les violences faites aux femmes pourraient-ils se transposer à d'autres groupes? Comment votre recette pourrait-elle fonctionner pour d'autres?

G. Sirois : Je crois que les gains des groupes de femmes sont transférables à d'autres secteurs, comme l'enfance ou les jeunes. Si on cherche à mieux desservir une population, et que l'expérience d'un autre groupe indique que l'approche utilisée est efficace, pourquoi ne pas emprunter certains de ses principes ou approches pour faire avancer les choses? Par exemple, le «par et pour » en matière de SEF est efficace dans notre secteur et celui de la petite enfance, pourquoi ne le serait-il pas ailleurs? Notre stratégie de concertation est exigeante en termes de déplacements il est vrai, mais elle nous a permis de mieux saisir les défis de la mise en œuvre de services et de trouver des solutions. Le modèle de la Ligne Fem'aide pourrait servir pour la population des ainés francophones, par exemple. Je ne prétends pas que nous avons "inventé " ces approches, mais nous les avons mises en place dans d'autres ministères; et à chaque fois qu'un groupe réussit à convaincre un ministère du bien-fondé de son approche, ça peut servir aux autres.

La compréhension des différentes formes d'oppression nous a bien servi aussi : comprendre la sienne en tant que femme, en 
tant que francophone ou en tant qu'immigrante, par exemple, permet de comprendre comment les individus réagissent à l'absence de pouvoir. Notre rôle en tant qu'intervenantes, c'est de redonner le pouvoir aux personnes marginalisées afin qu'elles puissent chercher à reprendre contrôle sur leur vie ou sur leur environnement.

Nous avons tous et toutes des droits, inscrits dans diverses conventions internationales ou chartes des droits, pas juste en matière d'égalité et de sécurité, mais en matière d'éducation, de travail ou de besoins de base. Faire connaitre ces droits, rassembler les personnes pour que ces droits soient respectés, voter pour un gouvernement qui va les respecter, tout ça fait partie de notre rôle d'intervenants sociaux, en plus de notre rôle d'aide et de soutien.

M.-L. Garceau: Vous auriez trois veux à formuler pour changer le monde, quels seraient$i l s$ ?

G. Sirois : Les droits à l'égalité des femmes, pas seulement sur papier, mais dans la vie de tous les jours. On mettrait fin à la violence faite aux femmes, à la dépendance financière, à l'exploitation sous toutes ses formes;

Un environnement sain, où les droits à l'eau et aux produits de la terre sont là pour tous et toutes;

De la compassion en abondance, plutôt que de la cupidité et de l'égoüsme...

\section{Notes}

«Action ontarienne contre la violence faite aux femmes s'engage à travailler à l'élimination de la violence faite aux femmes, par l'analyse, la formation et l'éducation et par la coordination de l'intervention des groupes qui luttent contre la violence faite aux femmes " (AOcVF, 2012, Mandat et mission, réf. du 19 janvier 2012, http://francofemmes.org/aocvf/index. cfm?Voir=menu\&Repertoire_No $=-1051436664 \& \mathrm{M}=1491$

2 Madame Sirois souligne ici l'implication de Lucie Brunet, chercheuse communautaire, et de Marie-Luce Garceau, chercheuse universitaire.

3 Gaëtane Pharand a été présidente d'AOcVF. 